\title{
INTEGRATION MAPS AND LOCAL EQUICONTINUITY OF SPECTRAL MEASURES
}

\author{
S. OKADA and W. J. RICKER \\ (Received 13 September 1999; revised 8 March 2000) \\ Communicated by P. G. Dodds
}

\begin{abstract}
One of the useful features of spectral measures which happen to be equicontinuous is that their associated integration maps are bicontinuous isomorphisms of the corresponding $L^{1}$-space onto their ranges. It is shown here that equicontinuity is not necessary for this to be the case; a somewhat weaker property suffices. This is of some interest in practice since there are many natural examples of spectral measures which fail to be equicontinuous.
\end{abstract}

2000 Mathematics subject classification: primary 47B15, 47B40, 47D30.

Since its conception the notion of a normal operator $T$ in a Hilbert space has been intimately connected with its resolution of the identity. This is a (unique) spectral measure $P$ defined on the Borel subsets of $\mathbb{C}$, with support the spectrum of $T$, such that $T$ is synthesized from the projections in the range of $P$ via a suitable integral. In the 1950's N. Dunford initiated the study of scalar-type spectral operators in a general Banach space. These are the analogues of normal operators in Hilbert space. As in the Hilbert space setting, the fundamental concept is again that of a spectral measure from which the given operator is synthesized; see [5], for example.

In the 1960's the theory of scalar-type spectral operators was extended to the setting of locally convex Hausdorff spaces (briefly, lcHs). But, new phenomena soon emerged in the non-normable setting which are simply not present in Banach spaces. Something as basic as the uniform boundedness of the range of a spectral measure, interpreted as equicontinuity in a lcHs, fails to hold in general, [9]. Since the uniform boundedness of a spectral measure in Banach spaces played such a crucial role in many of the arguments, most of the initial developments in the lc-setting dealt almost exclusively

Support of the Australian Research Council is gratefully acknowledged by the first author.

(C) 2000 Australian Mathematical Society $0263-6115 / 2000 \$ A 2.00+0.00$ 
with spectral measures which were assumed to be equicontinuous, $[1-4,6,18-21]$. As successful as the theory was for such spectral measures, it was also realized that it excluded many natural examples. Further investigations [12-15] revealed that in many arguments it is not so much the equicontinuity of the spectral measure $P$ which is relevant, but rather that its associated integration map $I_{P}: f \mapsto \int_{\Omega} f d P$, defined on the space $\mathscr{L}^{1}(P)$ of all $P$-integrable functions, should be a bicontinuous isomorphism of $\mathscr{L}^{1}(P)$ onto its range. Of course, equicontinuous spectral measures always have this property. Associated with $P$ is also the family of $X$-valued vector measures $\{P x: x \in X\}$, where $P x$ is specified via evaluation of the projections $P(E)$ at $x$ and $X$ denotes the underlying lcHs. These vector measures in turn induce the associated family of $X$-valued integration maps (one for each $x \in X$ ) given by $I_{P_{X}}: g \mapsto \int_{\Omega} g d(P x)$, defined on the space $\mathscr{L}^{1}(P x)$ of all $P x$-integrable functions. The importance of this family of maps $\left\{I_{P x}: x \in X\right\}$ lies with the fact that, under certain mild assumptions on the underlying $\mathrm{lcHs} X$, their ranges can be identified with the important class of cyclic subspaces of $X$ generated by $P$. It turns out that many features of the theory take on a simpler and more transparent form when these integration maps $\left\{I_{P_{X}}: x \in X\right\}$ are bicontinuous isomorphisms onto their ranges in $X$, a property which again is automatic if $P$ is equicontinuous.

The aim of this note is to investigate the connection between equicontinuity of $P$, the property of each integration map $I_{P x}$, for $x \in X$, being a bicontinuous isomorphism, and the property of the global integration map $I_{P}$ being a bicontinuous isomorphism. It is shown that $I_{P X}$ (for a given $x \in X$ ) is a bicontinuous isomorphism precisely when the restriction of $P$ to the cyclic space generated by $x$ is equicontinuous; we simply say that $P$ is locally equicontinuous at $x$ in this case. Moreover, it is shown that if $P$ is locally equicontinuous at every $x \in X$, then also the global integration map $I_{P}$ is necessarily a bicontinuous isomorphism. An example is given which shows that the converse of this statement is false in general. Examples of spectral measures $P$ for which $I_{P}$ fails to be a bicontinuous isomorphism have been known for some time, [15]. Of course, such a $P$ cannot be equicontinuous. We also exhibit a non-equicontinuous spectral measure $P$ which is both locally equicontinuous and for which $I_{P}$ is a bicontinuous isomorphism.

\section{Preliminaries}

Let $Y$ be a lcHs and $Y^{\prime}$ be its dual space, that is, the space of all $\mathbb{C}$-valued continuous linear functionals on $Y$. Given $y \in Y$ and $y^{\prime} \in Y^{\prime}$ we write $y^{\prime}(y)=\left\langle y, y^{\prime}\right\rangle$. Let $\mathscr{P}(Y)$ denote the family of all continuous seminorms on $Y$. The linear span of a subset $W$ in $Y$ is denoted by $\operatorname{sp}(W)$ and the closed linear span of $W$ in $Y$ by $\overline{\operatorname{sp}}(W)$. We denote the range of a linear operator $T$ by $\mathscr{R}(T)$. 
Let $\Sigma$ be a $\sigma$-algebra of subsets of a non-empty set $\Omega$. The characteristic function of each set $E \in \Sigma$ is denoted by $\chi_{E}$. By $\mathscr{L}^{0}(\Sigma)$ we denote the space of all C-valued, $\Sigma$-measurable functions on $\Omega$. The linear subspace of $\mathscr{L}^{0}(\Sigma)$ consisting of all $\Sigma$ simple functions on $\Omega$ is denoted by $\operatorname{sim}(\Sigma)$. A $\sigma$-additive set function $m: \Sigma \rightarrow Y$ is called a vector measure. The Orlicz-Pettis lemma [7, Theorem I.1.3] ensures that $m: \Sigma \rightarrow Y$ is $\sigma$-additive if and only if the set function $\left\langle m, y^{\prime}\right\rangle: \Sigma \rightarrow \mathbb{C}$ given by $\left\langle m, y^{\prime}\right\rangle(E)=\left\langle m(E), y^{\prime}\right\rangle$ for every $E \in \Sigma$ is $\sigma$-additive for each $y \in Y^{\prime}$.

Let $m: \Sigma \rightarrow Y$ be a vector measure. Let $[Y]_{m}$ denote the sequential closure of $\operatorname{sp}(m(\Sigma))$ in $Y$. It is always assumed that $[Y]_{m}$ has the relative topology from $Y$. A function $f \in \mathscr{L}^{0}(\Sigma)$ is called $m$-integrable if it is $\left\langle m, y^{\prime}\right\rangle$-integrable for each $y^{\prime} \in Y^{\prime}$ and if, given any $E \in \Sigma$, there is a unique vector $\int_{E} f d m$ in $Y$ satisfying $\left\langle\int_{E} f d m, y^{\prime}\right\rangle=\int_{E} f d\left\langle m, y^{\prime}\right\rangle$ for every $y^{\prime} \in Y^{\prime}$. Clearly every $\Sigma$-simple function is $m$-integrable. The vector space of all $m$-integrable functions is denoted by $\mathscr{L}^{1}(m)$. Let $q \in \mathscr{P}(Y)$. Define a seminorm $q(m)$ on $\mathscr{L}^{1}(m)$ by

$$
q(m)(f)=\sup _{E \in \Sigma} q\left(\int_{E} f d m\right), \quad f \in \mathscr{L}^{\mathrm{l}}(m) .
$$

Equip $\mathscr{L}^{1}(m)$ with the locally convex topology $\tau(m)$ defined by the family of seminorms $\{q(m): q \in \mathscr{P}(Y)\}$. This topology is the same as that defined in [7, Chapter II] and is called the mean convergence topology. The space $\operatorname{sim}(\Sigma)$ is sequentially $\tau(m)$ dense in $\mathscr{L}^{1}(m)$. In fact, this has been shown in [8, Theorem 2.2 and Theorem 2.4] with the additional assumption that $Y$ is sequentially complete. But, we do not actually need this assumption; see [10, Proposition 1.2].

A function $f \in \mathscr{L}^{1}(m)$ is called $m$-null if $\int_{E} f d m=0$ for every $E \in \Sigma$. We identify $\mathscr{L}^{1}(m)$ with its quotient space with respect to the closed subspace of all $m$-null functions. So, we can regard $\mathscr{L}^{1}(m)$ as a lcHs.

The vector measure $m$ is called closed if the subset $\Sigma(m)=\left\{\chi_{E}: E \in \Sigma\right\}$ of $\mathscr{L}^{1}(m)$ is complete with respect to the topology induced by $\tau(m)$, [7, page 71]. Whenever $[Y]_{m}$ is sequentially complete, the vector measure $m$ is closed if and only if $\mathscr{L}^{1}(m)$ is $\tau(m)$-complete [16, Theorem 2].

The integration map associated with $m$ is the map $I_{m}: \mathscr{L}^{1}(m) \rightarrow Y$ defined by

$$
I_{m}(f)=\int_{\Omega} f d m, \quad f \in \mathscr{L}^{1}(m) .
$$

Clearly $I_{m}$ is linear and continuous. Moreover, $\mathscr{R}\left(I_{m}\right) \subseteq[Y]_{m}$; see [10, page 347].

Let $X$ be a lcHs. The vector space of all continuous linear operators from $X$ into itself is denoted by $L(X)$. The space $L(X)$ equipped with the strong operator topology (that is, the topology of pointwise convergence on $X$ ) is a lcHs and is denoted by $L_{s}(X)$. The topology of $L_{s}(X)$ is generated by the family of seminorms

$$
q_{x}: T \mapsto q(T x), \quad T \in L(X),
$$


for all $x \in X$ and $q \in \mathscr{P}(X)$.

Let $P: \Sigma \rightarrow L_{s}(X)$ be a spectral measure. In other words, $P$ is $\sigma$-additive and multiplicative (that is, $P(E \cap F)=P(E) P(F)$ for all $E, F \in \Sigma)$, and $P(\Omega)$ equals the identity operator $I$ on $X$. The space $\mathscr{L}^{1}(P)$ is an algebra of functions (under pointwise operations) such that

$$
\int_{E} f g d P=P(E) I_{P}(f) I_{P}(g)=P(E) I_{P}(g) I_{P}(f), \quad E \in \Sigma,
$$

for all $f, g \in \mathscr{L}^{1}(P),\left[13\right.$, Corollary 2.1]. Therefore, the integration map $I_{P}$ : $\mathscr{L}^{1}(P) \rightarrow L_{s}(X)$ is a continuous, algebra homomorphism onto its range $\mathscr{R}\left(I_{P}\right)$, which is contained in $\left[L_{s}(X)\right]_{P}$. Moreover, $I_{P}$ is always injective because each $f \in I_{P}^{-1}(\{0\})$ satisfies $\int_{E} f d P=P(E) I_{P}(f)=0$ for every $E \in \Sigma$, that is, $f$ is $P$-null.

Let $x \in X$. Define a vector measure $P x: \Sigma \rightarrow X$ by $P x(E)=P(E) x$ for every $E \in \Sigma$. The integration map $I_{P_{X}}: \mathscr{L}^{1}(P x) \rightarrow X$ is also always injective because the multiplicativity of $P$ implies that

$$
\int_{E} f d(P x)=P(E) I_{P x}(f), \quad f \in \mathscr{L}^{1}(P x), E \in \Sigma .
$$

Furthermore, $\mathscr{L}^{1}(P) \subseteq \mathscr{L}^{1}(P x)$ and $\int_{E} g d(P x)=\left(\int_{E} g d P\right) x$ for every $g \in \mathscr{L}^{1}(P)$ and $E \in \Sigma$. The closed subspace $P(\Sigma)[x]=\overline{\mathrm{sp}}(P x(\Sigma))$ of $X$ is called the cyclic subspace generated by $x$; it always has the relative topology from $X$. Then $\mathscr{R}\left(I_{P_{x}}\right) \subseteq[X]_{P_{x}} \subseteq P(\Sigma)[x]$. Moreover, $\mathscr{R}\left(I_{P_{x}}\right)$ is dense in $P(\Sigma)[x]$ as it contains $I_{P x}(\operatorname{sim}(\Sigma))=\operatorname{sp}(P x(\Sigma))$.

\section{Locally equicontinuous spectral measures}

Throughout this section let $X$ be a lcHs and $P$ be an $L_{s}(X)$-valued spectral measure on a $\sigma$-algebra $\Sigma$ of subsets of a non-empty set $\Omega$.

The spectral measure $P$ is called equicontinuous if its range $P(\Sigma)$ is an equicontinuous subset of $L(X)$. If $X$ is quasi-barrelled, in particular, if $X$ is metrizable, then $P$ is necessarily equicontinuous [12, Proposition 2.5]. As noted in the introduction one of the most important features of equicontinuous spectral measures is the following one.

LEMMA 1. Suppose that the spectral measure $P$ is equicontinuous.

(i) For every $x \in X$, the integration map $I_{P x}: \mathscr{L}^{1}(P x) \rightarrow X$ is a bicontinuous isomorphism onto its range.

(ii) The integration map $I_{P}: \mathscr{L}^{1}(P) \rightarrow L_{s}(X)$ is a bicontinuous algebra isomorphism onto its range. 
ProOF. (i) The first part of the proof of Proposition 2.1 in [4] is still valid in our setting and establishes (i).

(ii) See [14, Lemma 1.11].

In this section we introduce the notion of locally equicontinuous spectral measures; they always satisfy (i) and (ii) of Lemma 1. Equicontinuous spectral measures are always locally equicontinuous, but the converse is not valid in general; a counterexample is given (see Example 8).

Let $x \in X$. Fix $E \in \Sigma$. The subspaces $\mathscr{R}\left(I_{P x}\right)$ and $P(\Sigma)[x]$ of $X$ are invariant for the operator $P(E)$. The restriction $\left.P(E)\right|_{\mathscr{R}\left(I_{P_{x}}\right)}$ of $P(E)$ to $\mathscr{R}\left(I_{P_{X}}\right)$ belongs to $L\left(\mathscr{R}\left(I_{P x}\right)\right)$. Similarly, the restriction $\left.P(E)\right|_{P(\Sigma)[x]}$ of $P(E)$ to $P(\Sigma)[x]$ is an operator

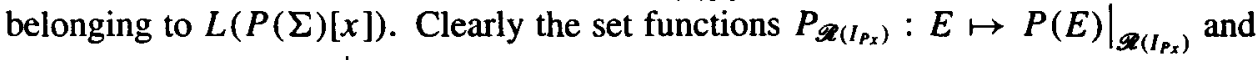
$P_{P(\Sigma)[x]}:\left.E \mapsto P(E)\right|_{P(\Sigma)[x]}$ are spectral measures with values in $L_{s}\left(\mathscr{R}\left(I_{P_{X}}\right)\right)$ and $L_{s}(P(\Sigma)[x])$ respectively.

The following result characterizes (for a fixed $x \in X$ ) the property (i) of Lemma 1 in terms of equicontinuity of the restriction of $P$ to certain invariant subspaces.

PROPOSITION 2. Let $P$ be a spectral measure and $x \in X$. The following statements are equivalent:

(i) The spectral measure $P_{P(\Sigma)[x]}: \Sigma \rightarrow L_{s}(P(\Sigma)[x])$ is equicontinuous.

(ii) The spectral measure $P_{\mathscr{R}\left(I_{p_{x}}\right)}: \Sigma \rightarrow L_{s}\left(\mathscr{R}\left(I_{P_{x}}\right)\right)$ is equicontinuous.

(iii) The integration map $I_{P_{x}}: \mathscr{L}^{\mathrm{l}}(P x) \rightarrow X$ is a bicontinuous isomorphism onto its range.

(iv) For each seminorm $q \in \mathscr{P}(X)$ there is a seminorm $r \in \mathscr{P}(X)$ such that

$$
q_{x}(P)(f) \leq r_{x}\left(I_{P} f\right), \quad f \in \mathscr{L}^{1}(P) .
$$

PROOF. (i) implies (iii). Given $q \in \mathscr{P}(X)$ there is $r \in \mathscr{P}(X)$ such that $q(P(E) y) \leq r(y)$ for each $E \in \Sigma$ and $y \in P(\Sigma)[x]$. We have used here the fact that every continuous seminorm on a subspace of $X$ is the restriction of some element (not necessarily unique) from $\mathscr{P}(X)$, whenever this subspace has the relative topology. From (1) and the inclusion $\mathscr{R}\left(I_{P_{X}}\right) \subseteq P(\Sigma)[x]$, we have

$$
\sup _{E \in \Sigma} q\left(\int_{E} f d(P x)\right)=\sup _{E \in \Sigma} q\left(P(E) I_{P x} f\right) \leq r\left(I_{P x} f\right)
$$

for every $f \in \mathscr{L}^{1}(P x)$. This implies that the continuous linear injection $I_{P_{x}}$ has a continuous inverse on its range $\mathscr{P}\left(I_{P x}\right)$.

(ii) implies (iii). This was established in the proof that (i) implies (iii).

(iii) implies (i). Given $E \in \Sigma$ define a linear operator $S_{E}: \mathscr{L}^{1}(P x) \rightarrow \mathscr{L}^{\mathrm{l}}(P x)$ by $S_{E}(f)=\chi_{E} f$ for every $f \in \mathscr{L}^{1}(P x)$. From the definition of the topology 
$\tau(P x)$ on $\mathscr{L}^{1}(P x)$ it follows that $\left\{S_{E}: E \in \Sigma\right\}$ is an equicontinuous subset of $L\left(\mathscr{L}^{1}(P x)\right)$. Again from (1) we see that $P(E)=I_{P x} S_{E}\left(I_{P_{x}}\right)^{-1}$ on $\mathscr{R}\left(I_{P_{x}}\right)$, for every $E \in \Sigma$. So, the family $\{P(E): E \in \Sigma\}$ restricted to $\mathscr{R}\left(I_{P_{x}}\right)$ is an equicontinuous subset of $L\left(\mathscr{R}\left(I_{P x}\right)\right)$. Since $\mathscr{R}\left(I_{P x}\right)$ is dense in $P(\Sigma)[x]$ it follows that $P_{P(\Sigma)[x]}(\Sigma)$ is equicontinuous in $L(P(\Sigma)[x])$.

(iii) implies (ii). This was established in the proof that (iii) implies (i).

(iii) implies (iv). Given $q \in \mathscr{P}(X)$ there is $r \in \mathscr{P}(X)$ such that

$$
q(P x)(g) \leq r\left(I_{P x} g\right), \quad g \in \mathscr{L}^{1}(P x) .
$$

If $f \in \mathscr{L}^{1}(P) \subseteq \mathscr{L}^{1}(P x)$, then it is routine to verify (by (1) again) that

$$
q(P x)(f)=q_{x}(P)(f) \text { and } r\left(I_{P x} f\right)=r_{x}\left(I_{P} f\right) .
$$

Thus (3) and (4) applied to $f$ yield (2).

(iv) implies (iii). Fix $q \in \mathscr{P}(X)$ and let $r \in \mathscr{P}(X)$ be as in (iv). We only need to verify that $\left(I_{P_{x}}\right)^{-1}$ is continuous on $\mathscr{R}\left(I_{P_{x}}\right)$. Let $\xi \in \mathscr{R}\left(I_{P_{x}}\right)$ and take $g \in \mathscr{L}^{1}\left(P_{X}\right)$ such that $\xi=I_{P_{x}} g$. Choose a sequence $\left\{g_{n}\right\}_{n=1}^{\infty} \subseteq \operatorname{sim}(\Sigma)$ such that $g_{n} \rightarrow g$ in $\mathscr{L}^{1}(P x)$ as $n \rightarrow \infty$. Then $I_{P x} g_{n} \rightarrow I_{P x} g$ in $X$ as $n \rightarrow \infty$. Since (4) holds for each $\Sigma$-simple function $f$, we have

$$
\begin{aligned}
q(P x)(g) & =\lim _{n \rightarrow \infty} q(P x)\left(g_{n}\right)=\lim _{n \rightarrow \infty} q_{x}(P)\left(g_{n}\right) \\
& \leq \lim _{n \rightarrow \infty} r_{x}\left(I_{P} g_{n}\right)=\lim _{n \rightarrow \infty} r\left(I_{P_{x}} g_{n}\right)=r\left(I_{P_{x}} g\right) .
\end{aligned}
$$

That is, $q(P x)\left(\left(I_{P_{x}}\right)^{-1} \xi\right) \leq r(\xi)$. Since $\xi \in \mathscr{R}\left(I_{P_{x}}\right)$ is arbitrary this establishes continuity of $\left(I_{P x}\right)^{-1}$ on its range.

The spectral measure $P$ is said to be locally equicontinuous if the restricted spectral measure $P_{P(\Sigma)[x]}: \Sigma \rightarrow L_{s}(P(\Sigma)[x])$ is equicontinuous for every $x \in X$. In particular, equicontinuous spectral measures are locally equicontinuous. The following result follows immediately from Proposition 2.

THEOREM 3. The spectral measure $P: \Sigma \rightarrow L_{s}(X)$ is locally equicontinuous if and only if for each $x \in X$ the integration map $I_{P_{x}}: \mathscr{L}^{1}(P x) \rightarrow X$ is a bicontinuous isomorphism onto its range.

Recall that a vector $x \in X$ is called a cyclic vector for $P$ if $X=P(\Sigma)[x]$ or, equivalently, if $X=\overline{\mathscr{R}\left(I_{P X}\right)}$.

COROLLARY 4. Suppose that there is a cyclic vector $x \in X$ for the spectral measure $P$. Then $P$ is equicontinuous if and only if the integration map $I_{P_{X}}$ is a bicontinuous isomorphism onto its range. 
The following result makes the connection between local equicontinuity of $P$ and continuity properties of the global integration map $I_{P}: \mathscr{L}^{1}(P) \rightarrow L_{s}(X)$.

THEOREM 5. If the spectral measure $P$ is locally equicontinuous, then the integration map $I_{P}: \mathscr{L}^{1}(P) \rightarrow L_{s}(X)$ is necessarily a bicontinuous linear and algebra isomorphism onto its range.

PROOF. Fix $x \in X$ and $q \in \mathscr{P}(X)$, which specify a typical seminorm $q_{x}$ generating the topology in $L_{s}(X)$. By Proposition 2(iv) there is $r \in \mathscr{P}(X)$ such that (2) is satisfied. Since $r_{x}$ is a continuous seminorm on $L_{s}(X)$ and $\mathscr{R}\left(I_{P}\right)$ has the relative topology from $L_{s}(X)$, this shows that the continuous injection $I_{P}$ has a continuous inverse on $\mathscr{R}\left(I_{P}\right)$.

REMARK. The seminorm $r$ given in the above proof may vary with $x$. Indeed, it is precisely this possible dependence of $r$ on $x$ (and $q$, of course) which allows for the possibility of $\left(I_{P}\right)^{-1}$ to be continuous without $P$ being equicontinuous; see Example 8 .

In view of Proposition 2 there arises the question of whether the inclusion $\mathscr{R}\left(I_{P_{x}}\right) \subseteq$ $P(\Sigma)[x]$ can be strict. Certainly if $X$ is metrizable, then $\mathscr{R}\left(I_{p_{x}}\right)$ is closed in $X$ and hence $R\left(I_{P_{X}}\right)=P(\Sigma)[x]$ for every $x \in X$. To see this, let $\left\{f_{n}\right\}_{n=1}^{\infty} \subseteq \mathscr{L}^{1}(P x)$ be a sequence such that $I_{P_{x}}\left(f_{n}\right) \rightarrow z$ as $n \rightarrow \infty$, for some $z \in X$. Then for each $E \in \Sigma$ it follows from (1) and the continuity of $P(E)$ that $I_{P_{x}}\left(\chi_{\varepsilon} f_{n}\right) \rightarrow P(E) z$ as $n \rightarrow \infty$. Accordingly, $I_{P_{x}}$ is $\Sigma$-converging in the sense of [11, page 516] and so Proposition 1.6 and Proposition 2.6 of [11] imply that $\mathscr{R}\left(I_{P_{x}}\right)$ is closed in the metrizable space $X$. However, the inclusion $\mathscr{R}\left(I_{P_{x}}\right) \subseteq P(\Sigma)[x]$ may be strict in the non-metrizable setting.

EXAMPLE 6. Let $X=\mathbb{C}^{[0,1]}$ be equipped with the product topology. Let $\Sigma$ be the $\sigma$ algebra of all Borel subsets of $\Omega=[0,1]$ and $P: \Sigma \rightarrow L_{s}(X)$ be the equicontinuous spectral measure defined by $P(E) x=\chi_{\varepsilon} x$ for every $E \in \Sigma$ and $x \in X$. For $x$ the constant function one on $\Omega$ it is routine to check that $\mathscr{R}\left(I_{P_{x}}\right)=\mathscr{L}^{0}(\Sigma) \varsubsetneqq X=$ $P(\Sigma)[x]$.

Since $\Sigma(P)=\left\{\chi_{E}: E \in \Sigma\right\}$ is always a closed set in $\mathscr{L}^{1}(P)$ it follows that if $I_{P}$ is a bicontinuous isomorphism onto its range, then $P(\Sigma)$ must be a closed subset in $\mathscr{R}\left(I_{P}\right) \subseteq L_{s}(X)$. In particular, if there exists $f \in \mathscr{L}^{1}(P) \backslash \Sigma(P)$ and a net $\left\{E_{\alpha}\right\} \subseteq \Sigma$ such that $P\left(E_{\alpha}\right) \rightarrow \int_{\Omega} f d P$ for the strong operator topology, then $I_{P}$ cannot be an isomorphism onto its range. For instance, let $X_{1}=L^{2}([0,1])$ be equipped with its weak topology, in which case $X_{1}$ is a quasicomplete lcHs. Let $\Omega=[0,1]$ and $\Sigma$ be the $\sigma$-algebra of all Borel subsets of $\Omega$. Then $P_{1}: \Sigma \rightarrow L_{s}\left(X_{1}\right)$ defined by $P_{1}(E): f \mapsto \chi_{E} f$, for each $f \in X_{1}$ and $E \in \Sigma$, is a (closed) spectral measure. It is shown in [17, pages 369-370] that there exists a sequence $\left\{E_{n}\right\}_{n=1}^{\infty} \subseteq \Sigma$ such that $P_{1}\left(E_{n}\right) \rightarrow(1 / 2) I$ in $\mathscr{R}\left(I_{P_{1}}\right)$. Accordingly, $\left(I_{P_{1}}\right)^{-1}$ is not continuous. 
The following example shows that the converse of Theorem 5 is not valid in general, that is, $I_{P}$ can be a bicontinuous isomorphism onto its range without being locally equicontinuous.

EXAMPLE 7. Let $P_{1}: \Sigma \rightarrow L_{s}\left(X_{1}\right)$ be as in the previous paragraph. Let $X_{2}=$ $L^{2}([0,1])$ be equipped with its usual norm $u: f \mapsto\left(\int_{0}^{1}|f(t)|^{2} d t\right)^{1 / 2}$. Define an equicontinuous spectral measure $P_{2}: \Sigma \rightarrow L_{s}\left(X_{2}\right)$ by $P_{2}(E): g \mapsto \chi_{E} g$, for each $g \in X_{2}$ and $E \in \Sigma$. Let $X$ denote the direct sum $X_{1} \oplus X_{2}$, equipped with the topology generated by the family of seminorms $\left\{\rho_{\psi}: \psi \in L^{2}([0,1])\right\}$ where

$$
\rho_{\psi}\left(f_{1} \oplus f_{2}\right)=\left|\left\langle f_{1}, \psi\right\rangle\right|+u\left(f_{2}\right), \quad f_{1} \oplus f_{2} \in X .
$$

Then $X$ is a quasicomplete lcHs. Define a (closed) spectral measure $P: \Sigma \rightarrow L_{s}(X)$ by $P(E)\left(f_{1} \oplus f_{2}\right)=P_{1}(E) f_{1} \oplus P_{2}(E) f_{2}$, for each $E \in \Sigma$ and $f_{1} \oplus f_{2} \in X$. Then $\mathscr{L}^{1}(P)=L^{\infty}([0,1])$ as vector spaces. Indeed, if $\varphi \in L^{\infty}([0,1])$, then

$$
\int_{E} \varphi d P: f_{1} \oplus f_{2} \mapsto \chi_{\varepsilon} \varphi f_{1} \oplus \chi_{E} \varphi f_{2}=\left(\int_{E} \varphi d P_{1}\right) f_{1} \oplus\left(\int_{E} \varphi d P_{2}\right) f_{2}
$$

for every $E \in \Sigma$ and $f_{1} \oplus f_{2} \in X$, which shows that $L^{\infty}([0,1]) \subseteq \mathscr{L}^{1}(P)$. Conversely, if $\varphi \in \mathscr{L}^{1}(P)$, then $\varphi \in \mathscr{L}^{1}(P x)$ for each $x \in X$. By considering elements of the form $x=0 \oplus f_{2}$, with $f_{2} \in X_{2}$, and noting that $P(E) x=0 \oplus P_{2}(E) f_{2}$ is an element of the closed $P$-invariant subspace $\{0\} \oplus X_{2} \simeq X_{2}$, for each $E \in \Sigma$, it follows that $\varphi \in \mathscr{L}^{\prime}\left(P_{2} f_{2}\right)$ for each $f_{2} \in X_{2}$. But, $\mathscr{L}^{\prime}\left(P_{2} f_{2}\right)=\left\{g \in \mathscr{L}^{0}(\Sigma): g f_{2} \in L^{2}([0,1])\right\}$ with $\int_{E} g d\left(P_{2} f_{2}\right)=\chi_{E} g f_{2}$ for each $E \in \Sigma$. Choosing $E=[0,1]$ we see that $\varphi f_{2} \in L^{2}([0,1])$ for all $f_{2} \in L^{2}([0,1])$ which implies that $\varphi \in L^{\infty}([0,1])$.

The seminorms generating the topology $\tau\left(P_{2}\right)$ in $\mathscr{L}^{1}\left(P_{2}\right)$ are of the form

$$
u_{\xi}\left(P_{2}\right): \varphi \mapsto \sup _{E \in \Sigma} u\left(\left(\int_{E} \varphi d P_{2}\right) \xi\right), \quad \varphi \in L^{\infty}([0,1])=\mathscr{L}^{1}\left(P_{2}\right),
$$

for $\xi \in L^{2}([0,1])$. The seminorms generating $\tau\left(P_{1}\right)$ are of the form

$$
q_{\psi, \xi}\left(P_{1}\right): \varphi \mapsto \sup _{E \in \Sigma}\left|\left\langle\left(\int_{E} \varphi d P_{1}\right) \xi, \psi\right\rangle\right|, \quad \varphi \in L^{\infty}([0,1])=\mathscr{L}^{1}\left(P_{1}\right),
$$

for arbitrary $\psi, \xi \in L^{2}([0,1])$. By the Cauchy-Schwarz inequality it follows that

$$
q_{\psi, \xi}\left(P_{1}\right)(\varphi) \leq u(\psi) \cdot u_{\xi}\left(P_{2}\right)(\varphi), \quad \varphi \in L^{\infty}([0,1]),
$$

for all $\psi, \xi \in L^{2}([0,1])$.

Let $\left\{\varphi_{\alpha}\right\} \subseteq L^{\infty}([0,1])$ be a net such that $I_{P}\left(\varphi_{\alpha}\right) \rightarrow 0$ in $\mathscr{R}\left(I_{P}\right)$. To show $\left(I_{P}\right)^{-1}$ is continuous we need to verify that $\varphi_{\alpha} \rightarrow 0$ in $\mathscr{L}^{1}(P)$. By considering the elements 
$x=0 \oplus g \in X$, where $g \in X_{2}$, it follows from $I_{P}\left(\varphi_{\alpha}\right) \rightarrow 0$ in $\mathscr{R}\left(I_{P}\right)$ that $I_{P_{2}}\left(\varphi_{\alpha}\right) \rightarrow 0$ in $\mathscr{R}\left(I_{P_{2}}\right) \subseteq L_{s}\left(X_{2}\right)$. A typical seminorm generating $\tau(P)$ is of the form (for $\left.\varphi \in \mathscr{L}^{1}(P)\right)$

$$
\begin{aligned}
\left(\rho_{\psi}\right)_{\xi_{1} \oplus \xi_{2}}(P)(\varphi) & =\sup _{E \in \Sigma} \rho_{\psi}\left(\left(\int_{E} \varphi d P_{1}\right) \xi_{1} \oplus\left(\int_{E} \varphi d P_{2}\right) \xi_{2}\right) \\
& \leq \sup _{E \in \Sigma}\left|\left\langle\left(\int_{E} \varphi d P_{1}\right) \xi_{1}, \psi\right\rangle\right|+\sup _{E \in \Sigma} u\left(\left(\int_{E} \varphi d P_{2}\right) \xi_{2}\right) \\
& =q_{\psi, \xi_{1}}\left(P_{1}\right)(\varphi)+u_{\xi_{2}}\left(P_{2}\right)(\varphi),
\end{aligned}
$$

for some $\psi \in L^{2}([0,1])$ and $\xi_{1} \oplus \xi_{2} \in X$. Then (5) implies that

$$
\left(\rho_{\psi}\right)_{\xi_{1} \oplus \xi_{2}}(P)(\varphi) \leq u(\psi) \cdot u_{\xi_{1}}\left(P_{2}\right)(\varphi)+u_{\xi_{2}}\left(P_{2}\right)(\varphi), \quad \varphi \in \mathscr{L}^{1}(P) .
$$

Since $P_{2}$ is equicontinuous it follows that $\left(I_{P_{2}}\right)^{-1}$ is continuous (see Lemma 1). But, $I_{P_{2}}\left(\varphi_{\alpha}\right) \rightarrow 0$ in $\mathscr{R}\left(I_{P_{2}}\right) \subseteq L_{s}\left(X_{2}\right)$ and so $\varphi_{\alpha} \rightarrow 0$ in $\mathscr{L}^{1}\left(P_{2}\right)$. In particular, $u_{\xi_{1}}\left(P_{2}\right)\left(\varphi_{\alpha}\right) \rightarrow 0$ and $u_{\xi_{2}}\left(P_{2}\right)\left(\varphi_{\alpha}\right) \rightarrow 0$ for each $\xi_{1}, \xi_{2} \in L^{2}([0,1])$, and we see from (6) that $\left(\rho_{\psi}\right)_{\xi_{1} \oplus \xi_{2}}(P)\left(\varphi_{\alpha}\right) \rightarrow 0$. This shows that $\varphi_{\alpha} \rightarrow 0$ in $\mathscr{L}^{1}(P)$ and hence, $\left(I_{P}\right)^{-1}$ is continuous. Accordingly, $I_{P}$ is a bicontinuous isomorphism of $\mathscr{L}^{1}(P)$ onto its range.

To see that $P$ is not locally equicontinuous we argue as follows. As noted earlier $\left(I_{P_{1}}\right)^{-1}$ is not continuous on $\mathscr{R}\left(I_{P_{1}}\right) \subseteq L_{s}\left(X_{1}\right)$ and so by Theorem 5 there must exist $f \in X_{1}$ such that $\left(I_{P_{1} f}\right)^{-1}$ is not continuous from $\mathscr{R}\left(I_{P_{1} f}\right) \subseteq X_{1}$ onto $\mathscr{L}^{1}\left(P_{1} f\right)$. Then $x=f \oplus 0 \in X$ has the property that $\left(I_{P_{X}}\right)^{-1}$ is not continuous from $\mathscr{R}\left(I_{P_{x}}\right) \subseteq X$ onto $\mathscr{L}^{1}(P x)$, where we have used the easily verified facts that $\mathscr{L}^{1}(P x) \simeq \mathscr{L}^{1}\left(P_{1} f\right)$ and $\mathscr{R}\left(I_{P_{x}}\right)=\mathscr{R}\left(I_{P_{1} f}\right) \oplus\{0\}$.

It may be of interest to note that $\mathscr{L}^{1}(P)$ is actually $\tau(P)$-complete. Indeed, from the various definitions and inequalities above we see easily that

$$
u_{\xi}\left(P_{2}\right)(\varphi)=\left(\rho_{\psi}\right)_{0 \oplus \xi}(P)(\varphi), \quad \varphi \in L^{\infty}([0,1]),
$$

for each $\xi \in L^{2}([0,1])$ and $\psi \in L^{2}([0,1])$. Since $\mathscr{L}^{1}(P)=L^{\infty}([0,1])=\mathscr{L}^{1}\left(P_{2}\right)$ as vector spaces, the previous equality and (6) show that $\mathscr{L}^{1}(P)$ and $\mathscr{L}^{1}\left(P_{2}\right)$ are isomorphic as lcHs. But, $\mathscr{L}^{1}\left(P_{2}\right)$ is complete [14, Proposition 3.16], as $P_{2}$ is a closed measure [14, Proposition 3.9]) and $\left[L_{s}\left(X_{2}\right)\right]_{P_{2}}$ is sequentially complete. We know that $\left[L_{s}\left(X_{2}\right)\right]_{P_{2}}$ is sequentially complete because the space $L_{s}\left(X_{2}\right)$ is quasicomplete. Consequently, also $\mathscr{L}^{1}(P)$ is complete. Since $I_{P}$ is a bicontinuous isomorphism of $\mathscr{L}^{1}(P)$ onto its range it follows that $\mathscr{R}\left(I_{P}\right)$ is a complete subspace of $L_{s}(X)$.

We conclude with an example of a spectral measure $P$ which is not equicontinuous, but which is locally equicontinuous. In particular, $I_{P}$ is then also a bicontinuous isomorphism onto its range (see Theorem 5). 
EXAMPLE 8. Let $\Omega$ be an infinite set and let $X$ denote the space $c_{00}(\Omega)$ of all $\mathbb{C}$ valued functions $x$ on $\Omega$ such that $x$ vanishes outside a finite subset of $\Omega$. Let $Y$ denote the space $\ell^{1}(\Omega)$ of all $\mathbb{C}$-valued functions $y$ on $\Omega$ satisfying $\Sigma_{\omega \in \Omega}|y(\omega)|<\infty$. Equip $X$ with the weakest topology $\sigma(X, Y)$ making each functional $y \in Y$ continuous on $X$, where $\langle x, y\rangle=\sum_{\omega \in \Omega} x(\omega) y(\omega)$ for each $x \in X$. Let $\ell^{\infty}(\Omega)$ denote the space of all bounded $\mathbb{C}$-valued functions on $\Omega$.

Let $f$ be a $\mathbb{C}$-valued function on $\Omega$. Define a linear operator $M_{f}: X \rightarrow X$ by $M_{f}: x \mapsto x f$ for every $x \in X$. Then $\left\langle M_{f} x, y\right\rangle=\sum_{\omega \in \Omega} x(\omega)(y f)(\omega)$ for every $x \in X$ and $y \in Y$. Hence, $M_{f}$ is continuous if and only if $y f \in Y$ for every $y \in Y$, that is, if and only if $f \in \ell^{\infty}(\Omega)$.

Let $\Sigma$ be a non-trivial $\sigma$-algebra of subsets of $\Omega$ (that is, $\Sigma$ contains infinitely many elements). Define a set function $P: \Sigma \rightarrow L_{s}(X)$ by $P(E)=M_{\chi_{E}}$ for each $E \in \Sigma$. It is routine to verify that $P$ is a spectral measure, that $\mathscr{L}^{1}(P)=\mathscr{L}^{0}(\Sigma) \cap \ell^{\infty}(\Omega)$ and that $\int_{E} f d P=P(E) M_{f}$ for every $f \in \mathscr{L}^{1}(P)$ and $E \in \Sigma$.

Fix $x \in X$ and let $x^{-1}(\mathbb{C} \backslash\{0\})=\left\{\omega_{1}, \cdots, \omega_{n}\right\}$. A typical seminorm $q$ generating the topology of $X$ is of the form $q(z)=|\langle z, y\rangle|, z \in X$, for some $y \in Y$. Define $r \in \mathscr{P}(X)$ by

$$
r(z)=\sum_{k=1}^{n}\left|\left\langle z, y\left(\omega_{k}\right) \chi_{\left(\omega_{k}\right)}\right\rangle\right|, \quad z \in X .
$$

Then it is easily verified that

$$
q_{x}(P)(f)=\sup _{E \in \Sigma}\left|\sum_{k=1}^{n} f\left(\omega_{k}\right) x\left(\omega_{k}\right) y\left(\omega_{k}\right) \chi_{E}\left(\omega_{k}\right)\right| \leq r_{x}\left(I_{P} f\right),
$$

for every $f \in \mathscr{L}^{1}(P)$. Proposition 2 shows that the integration map $I_{P x}$ is a bicontinuous isomorphism onto its range. Since $x \in X$ is arbitrary it follows from Theorem 3 that $P$ is locally equicontinuous. Then Theorem 5 ensures that $I_{P}$ is a bicontinuous isomorphism of $\mathscr{L}^{1}(P)$ onto its range.

Finally, to see that $P$ fails to be equicontinuous we refer to Example 1(iv) and Proposition 3 of [9].

It may be in interest to note that $\mathscr{L}^{1}(P)$ is typically not complete for this example. Indeed, suppose that $\Omega$ is uncountable, that $\Sigma$ contains all singleton sets $\{\omega\}$, for $\omega \in \Omega$, but $\Sigma \neq 2^{\Omega}$. Then there exists an infinite subset $F \subseteq \Omega$ which is not an element of $\Sigma$. Let $\mathscr{F}$ denote the family of all finite subsets of $F$ directed by inclusion. Then $\{P(E)\}_{E \in \mathscr{F}} \subseteq \mathscr{R}\left(I_{P}\right)$ is a net which converges to $M_{\chi_{F}}$ in $L_{s}(X)$. Since $\chi_{F} \notin \mathscr{L}^{1}(P)$ we see that $\{P(E)\}_{E \in \mathscr{F}}$ is a Cauchy net in $\mathscr{R}\left(I_{P}\right)$ having no limit in $\mathscr{R}\left(I_{P}\right)$. But, $I_{P}$ is a bicontinuous isomorphism of $\mathscr{L}^{1}(P)$ onto its range and so $\mathscr{L}^{1}(P)$ cannot be complete.

REMARK. Example 7 and Example 8 both provide spectral measures $P$ which are not equicontinuous, but such that $I_{P}$ is a bicontinuous isomorphism of $\mathscr{L}^{1}(P)$ onto its range. This answers a question posed in [14, page 13]. 


\section{References}

[1] P. G. Dodds and B. de Pagter, 'Orthomorphisms and Boolean algebras of projections', Math. Z. 187 (1984), 361-381.

[2] - 'Algebras of unbounded scalar-type spectral operators', Pacific J. Math. 130 (1987), 41-74.

[3] P. G. Dodds, B. de Pagter and W. J. Ricker, 'Reflexivity and order properties of scalar-type spectral operators in locally convex spaces', Trans. Amer. Math. Soc. 293 (1986), 355-380.

[4] P. G. Dodds and W. J. Ricker, 'Spectral measures and the Bade refiexivity theorem', J. Funct. Anal. 61 (1985), 136-163.

[5] N. Dunford and J. T. Schwartz, Linear operators III: Spectral operators (Wiley-Interscience, New York, 1971).

[6] C. Ionescu-Tulcea, 'Spectral operators on locally convex spaces', Bull. Amer. Math. Soc. 67 (1961), $125-128$.

[7] I. Kluvánek and G. Knowles, Vector measures and control systems (North Holland, Amsterdam, 1976).

[8] D. R. Lewis, 'Integration with respect to vector measures', Pacific J. Math. 33 (1970), 157-165.

[9] S. Okada and W. J. Ricker, 'Spectral measures which fail to be equicontinuous', Period. Math. Hungar. 28 (1994), 55-61.

[10] - 'Vector measures and integration in non-complete spaces', Arch. Math. (Basel) 63 (1994), $334-353$.

[11] , 'The range of the integration map of a vector measure', Arch. Math. (Basel) 64 (1995), $512-522$.

[12] - 'Continuous extensions of spectral measures', Colloq. Math. 71 (1996), 115-132.

[13] - 'Spectral measures and automatic continuity', Bull. Belgian Math. Soc. 3 (1996), 267-279.

[14] - 'Boolean algebras of projections and ranges of spectral measures', Dissertationes Math. 365 (1997), 33pp.

[15] W. J. Ricker, 'Boolean algebras of projections and spectral measures in dual spaces', Operator Theory: Adv. Appl. 43 (1990), 289-300.

[16] —, 'Completeness of the $L^{1}$-space of closed vector measures', Proc. Edinburgh Math. Soc. 33 (1990), 71-78.

[17] _ 'The sequential closedness of $\sigma$-complete Boolean algebras of projections', J. Math. Anal. Appl. 208 (1997), 364-371.

[18] W. J. Ricker and H. H. Schaefer, 'The uniformly closed algebra generated by a complete Boolean algebra of projections', Math. Z. 201 (1989), 429-439.

[19] H. H. Schaefer, 'Spectral measures in locally convex algebras', Acta Math. 107 (1962), 125-173.

[20] A. Shuchat, 'Vector measures and scalar operators in locally convex spaces', Michigan Math. J. 24 (1977), 303-310.

[21] B. Walsh, 'Structure of spectral measures on locally convex spaces', Trans. Amer. Math. Soc. 120 (1965), 295-326.

\section{School of Mathematics}

University of New South Wales

Sydney, NSW 2052

Australia

e-mail: okada@maths.unsw.edu au

e-mail: werner@maths.unsw.edu.au 